\title{
Primary Immunodeficiency Diseases in Costa Rican Adults. A Cross-Sectional Study
}

\author{
Alberto Alfaro Murillo \\ University of Costa Rica \\ Christian Schauer \\ Costa Rica Institute of Technology \\ Milena Castro Mora \\ University of Costa Rica \\ Nayely Marquez Salazar \\ Mandarina Labs, San José \\ Mauricio Alfonso Herrera Morice \\ Hospital Dr. Rafael Ángel Calderón Guardi \\ María Paz León Bratti \\ Laboratory of Clinical Immunology
}

María del Rosario Espinoza Mora ( $\nabla$ maria.espinoza@ucr.ac.cr )

University of Costa Rica https://orcid.org/0000-0002-3374-8563

\section{Research Article}

Keywords: primary immunodeficiency diseases, common variable immunodeficiency, adult, prevalence, Costa Rica, Bayes Theorem

Posted Date: October 25th, 2021

DOI: https://doi.org/10.21203/rs.3.rs-922689/v1

License: (c) (i) This work is licensed under a Creative Commons Attribution 4.0 International License.

Read Full License 


\section{Abstract}

Purpose: Up to date, neither in Costa Rica nor in Central America public registries regarding the prevalence and characterization of primary immunodeficiency diseases (PID) in adult patients exist. The aim of this study was to characterize the clinical features of patients diagnosed with primary and idiopathic immune disorders treated in two specialized immunodeficiency clinics in Costa Rica.

Methods: A cross-sectional study was conducted between 2017 and 2018, including 137 adult patients diagnosed with PID at two public hospitals in Costa Rica. To estimate a robust prevalence of PID in Costa Rica, a binomial model using a hyperparameter with a Poisson distribution was implemented in WinBUGS to fit a Bayesian model to approach a posterior distribution for the prevalence estimation.

Results: Patients with PID showed a heterogeneous distribution and clinical course. Prevalence estimated resulted in a mean of 3.35 patients per 100,000 inhabitants in Costa Rica by 2018, with a $95 \%$ confidence interval of 2.98 to 4.15 patients per 100,000 inhabitants. A higher frequency of PID was observed in women and patients between 30 to 59 years of age. Humoral immunodeficiencies were predominant and most common immunodeficiency manifestations were recurrent infections and atopic syndrome. Most frequent comorbidities were dyslipidemia, acid peptic disease and hypertension.

Conclusions: In this cohort, we found different clinical manifestations in comparison to other regions, highlighting the importance of a prompt diagnose of these entities in adults. Metabolic diseases were identified as common comorbidities in this group. Appropriate prevalence estimations can address diagnostic strategies.

\section{Introduction}

Primary immunodeficiency diseases (PID) are rare disorders of the innate and adaptive immunity, usually diagnosed early in life, although some defects present themselves in adulthood. About 400 clinical entities have been described as PID. They are characterized by susceptibility to frequent infections, autoimmune disorders, autoinflammatory syndromes, hemophagocytosis, lymphoproliferative disorders and malignancies, among others [1-5].

In adults, PID are diagnosed in three different scenarios, patients who develop immunodeficiency during adulthood, adult patients with pediatric PID undiagnosed or not treated during childhood and pediatric PID patients reaching adulthood [6]. The most diagnosed PID in adults are antibody deficiencies as IgA deficiency, common variable immunodeficiency (CVID), IgG subclass deficiency and specific antibody deficiency. Adults may also present less severe combined immunodeficiencies such as late onset combined immunodeficiency (LOCID), immunodeficiency with thymoma (Good's syndrome), and idiopathic $\mathrm{CD} 4^{+} \mathrm{T}$ cell lymphopenia, among others. On the other hand, chronic granulomatous disease, hyper-IgE syndromes, complement deficiencies, familial hemophagocytic syndromes, and primary neutropenia belong to the group of classical childhood PID with patients that reach adulthood [7-10]. 
The prevalence of PID in the United States of America is estimated to be approximately 1 in 1200 live births, with exception of selective IgA deficiency (SIgAD), the most common PID which affects approximately 1 in 300 to 1 in 500 people [11].The Latin American Association for Immunodeficiencies (LASID) reported in 20187633 registered cases of 105 different PID in both, adult and pediatric patients, distributed in 128 hospitals of 16 countries. In Costa Rica only pediatric patients are registered in this database as the only participating center was the National Children's Hospital reporting 117 patients [12]. This study implemented the first PID national registry in Central America for patients above 18 years (age in which pediatric care in Costa Rica concludes). Two specialized clinics for adult immunodeficiencies exist in the country, affiliated to the internal medicine departments of the national university hospitals, Dr. Rafael Angel Calderón Guardia Hospital and México Hospital.

\section{Methods}

This study consists of a cross-sectional analysis including all PID cases in patients over 18 years of age who were controlled ambulatory at specialized immunology clinics in Costa Rica from 1985 to 2018. Data were collected in 2018 from clinical records and laboratory databases. To calculate the prevalence proportion, the number of registries with a positive diagnosis of PID and the adult population from the national projections 2018 of the Central American Population Center, University of Costa Rica, were employed.

A binomial model using a hyperparameter with a Poisson distribution was implemented in WinBUGS to fit a Bayesian model to approach a posterior distribution for the prevalence estimation. Additional efforts were undertaken to identify all sources of registries; however, access was not granted for all possible clinical records, and therefore a distribution of the prevalence is considered Poisson accounting for a lower impact of the selection bias. Markov Chain Monte Carlo methods were used for the simulation of the prevalence estimations. Descriptive analysis was performed using R software, to obtain a wider perspective of the clinical characteristics of the patients and provides a qualitative version of the prevalence proportion. Origin of residence was considered to obtain a spatial distribution of the prevalence of PID.

Sensibility analysis was undertaken by increasing the assumption of the prevalence by $50 \%$ based on the distribution of patients by clinic. The distribution of patients by clinic was uniform, therefore a model with a $50 \%$ elevation in the number of cases was included in the proportion estimation to obtain a new distribution and to evaluate the respective increase in the patients per 100,000 inhabitants.

The study was authorized by the institutional bioethics committee and the Health Ministry of Costa Rica.

\section{Results}

The study registered a total of 137 patients, 95 patients from Dr. Rafael Angel Calderón Guardia Hospital and 42 patients from México Hospital, with the following PID: CVID, X-linked agammaglobulinemia (XLA), 
selective IgA deficiency, IgG subclasses deficiency, selective IgM deficiency, specific polysaccharide antibody deficiency (SPAD), primary complement deficiencies (C3, C4, C1q, hereditary angioedema type I and type II), ataxia telangiectasia, Job syndrome, idiopathic neutropenia, chronic mucocutaneous candidiasis, chronic granulomatous disease, idiopathic $\mathrm{CD} 4^{+} \mathrm{T}$ cell lymphopenia, autoimmune polyglandular syndrome type I (APS-1), mendelian susceptibility to mycobacteria and susceptibility to herpes viruses.

Due to the small size and high heterogeneity of the sample, and to facilitate further statistical analysis the entities were grouped in humoral disorders (complement deficiencies and antibody deficiencies), cellular disorders (primary neutropenia and lymphopenia) and others syndromic entities and mixed disorders.

Most of the patients were in a range of age between 30 and 59 years and $61.31 \%$ were female. The of them presented comorbidities, most frequently dyslipidemia (29.20\%) and acid peptic diseases (18.98\%) (Table 1).

The distribution of PID patients was not homogenous between the provinces of Costa Rica. (Figure 1)

An elevated number of cases per 100,000 habitants was observed in the provinces representing the metropolitan area (San José 5.55/100,000; Cartago 3.26/100,000); Heredia 3.23/100,000). Two density posterior distributions from a Poisson-Binomial Bayesian model for calculating the prevalence of PID in the population were effect for two situations. a) A liberal estimation of the prevalence assuming that almost all PID patients had been included in the study. The estimated prevalence resulted in a mean of 3.35 patients per 100,000 inhabitants in Costa Rica by 2018, with a $95 \%$ confidence interval of 2.98 to 4.15 patients per 100,000 inhabitants. b) A conservative estimation considering a $50 \%$ increment in the number of cases diagnosed with PID. In this estimation the mean prevalence was 5.29 patients per 100,000 inhabitants and its credible interval starts at 4.59 up to 6.04 patients per 100,000 inhabitants in Costa Rica. (Figure 2) The comparison of prevalence from both models results in a difference of 1.94 cases per 100,000 people, representing the putative level of underdiagnosis in PID in Costa Rica.

Infectious manifestation led in $59.12 \%$ of the patients to diagnosis or occurred during follow-up (Table 2). The most common pathogens were encapsulated bacteria, chickenpox, Epstein Barr virus, Candida albicans and Trichophyton rubrum. Infections with Cryptococcus neoformans and Aspergillus sp. were observed predominantly in patients with idiopathic $\mathrm{CD} 4^{+}$lymphopenia. In cases of mendelian susceptibility to mycobacteria, infections with Mycobacterium leprae and Mycobacterium tuberculosis were present (data not shown).

29.93\% of patients manifested one or more autoimmune disorders (Table 2). In this cohort, the PID associated with autoimmune diseases were SIgAD, selective IgM deficiency, C3 and C4 primary deficiencies, CVID and mixed immunodeficiencies. Furthermore, $48.18 \%$ of patients presented atopic manifestations as allergic rhinitis, asttuehma, atopic dermatitis and urticaria. The comorbidity of atopic syndromes was predominantly in SPAD (57.14\%), SIgAD (66.67\%) and CVID (57.89\%), only $7.30 \%$ of 
patients possessed neoplasms. This kind of association was found in primary complement deficiencies, SPAD, idiopathic neutropenia, CVID and mixed immunodeficiencies.

The clinical manifestations at the onset were chronic sinusitis in the first place (32.85\%), followed by recurrent pneumonia (30.66\%) and recurrent pharyngoamigdalitis (19.71\%). The most frequent secondary complications of PID were chronic sinusitis $(29.20 \%)$, recurrent bronchitis $(14.60 \%)$ and bronchiectasis (13.87\%).

$17.52 \%$ of patients were receiving replacement therapy with intravenous immunoglobulin (IVIG). This treatment was administrated to patients with XLA (in $100 \%$ of cases), CVID (in $78.95 \%$ of cases), HIES (in $100 \%$ of cases), and IgG subclasses deficiency (in $20.00 \%$ of cases). Only $14.60 \%$ of patients required oral antimicrobial prophylaxis, mainly in case of CVID.

\section{Discussion}

This study presents the first national registry of primary immunodeficiencies in adult patients in a Central American country. The PID records in Latin America are not systematic, therefore their frequencies are estimated to be higher than those reported [13]. In adults, PID should be considered in presence of recurrent or persistent infections of unusual severity or rapid progression, affecting multiple sites or being caused by opportunistic or unusual pathogens, leukopenia, history of parental consanguinity and positive family anamnesis [14]. However, other findings that should guide the diagnosis are more than one pneumonic episode or one or more serious infections in the past, frequent use of antibiotics and suboptimal response to treatment, non-surgical absence of adenoids, recurrent skin, soft tissue and deep organs abscesses, recurrent oral and genital ulcers, recurrent eczema, diffuse presence of warts or molluscum contagiosum, non-infectious granulomas, idiopathic bronchiectasis, persistent lymphopenia or neutropenia, idiopathic hypereosinophilia, IgE higher than $2000 \mathrm{IJ} / \mathrm{mL}$, autoimmune or allergic diseases, chronic diarrhea, characteristic facies, skeletal abnormalities, neurological symptoms and hearing loss $[14,15] 12$.

In this study, PID diagnostics were made under the criterion of clinical immunologists, based on the phenotypic analysis of each case, the laboratory tests available in public hospitals, and genetic studies in some cases. Similar to many Latin American countries, Costa Rica has limitations in PID diagnosis, as no genomic studies can be provided to confirm all suspected cases, nor functional immunological tests are available always at the public health system.

The largest number of patients diagnosed with PID came from the central urban region, which is expected as the majority of the Costa Rican population is concentrated in this area. A statistical analysis of the frequency of PID according to the urban and rural areas was not possible as a clear differentiation between these populational zones is not well defined in Costa Rica. The distribution by province of origin to establish whether there is any genetic pattern justifying variation in prevalence remains unclear. The high heterogeneity in PID distribution per province might be related to the presence of specialized clinics only in urban areas. 
In relation to the aimed national prevalence estimation of this study, there were two distributions modeled with a Poisson pattern, given the condition of a rare disease under study. A parameter $p$, representing the proportion of people that has been diagnosed with PID in Costa Rica, is denoted as our estimation of interest. One of these models is based only in verified diagnosed cases, whereas the other model describes a scenario including hidden cases related to underdiagnosed PID patients considering a possible underestimation. This model considers patients that may not have been diagnosed due to different possible reasons, as for example living too far from specialized clinics or lack of expertise diagnosing PID in physicians not sufficiently trained in immunology.

Female patients showed a higher prevalence of PID in this study. PID registries in Latin America are limited and distribution data for these pathologies according to sex and geographic area are in general controversial. US registries show higher prevalence of any kind of PID female sex [16] In contrary, German records report higher prevalence in men, similar as in Iran and the United Kingdom [17] The Swiss register shows an equitable prevalence between the two sexes [18] It should be noted that, although a higher PID trend is documented in the male sex, many of the registries are intertwined with pediatric populations, where X-linked conditions are common. In records involving only adult populations, the PID prevalence tends to be similar in both sexes [19].

The most common PID in this registry was idiopathic neutropenia due to the cohort of Hospital Dr. Calderón Guardia patients, while in the cohort of Hospital México the most frequently diagnosed PID was CVID. All neutropenic patients had histological bone marrow study and cytogenetic analysis to exclude hematological malignancies and aplastic syndromes.

Unlike to globally published data, in which the most common PID is SIgAD $[9,20]$, our study shows that this disorder does not seem to prevail in Costa Rica. However, excluding idiopathic neutropenia, the most prevalent diagnoses were humoral PID, that complies with the epidemiology described in other countries $[3,21]$.

In this study, PID patients frequently associated atopic manifestations, mainly of the antibody disorders subgroup. Previously, a close relationship between PID and atopic syndrome has been well documented, possibly mediated through non-lgE related Th1 and Th2 responses [22, 23].

The most common comorbidity detected in this cohort was dyslipidemia, accompanied by hepatic steatosis in some cases. It is unclear whether metabolic diseases could be another consequence of immune incompetence, treatment or an independent factor. No clinical studies provide information about incidence of metabolic and cardiovascular disease in the adult PID population up to now. Efforts in this regard need to be made, as metabolic syndrome may add comorbidity to immunodeficient patients, exacerbating the accompanying inflammatory state.

Autoimmune diseases were common in this registry, related in the first place to antibody deficiencies, which has been documented by previous studies [24] Patients with primary complement disorders also show autoimmune disorders. Deficiency of early components in the classic complement pathway favors 
the development of systemic autoimmune diseases, principally systemic lupus erythematosus [25]. In this study, 12 cases with primary complement defects and concomitant systemic lupus erythematosus (SLE) were documented in this study. These patients remained asymptomatic but showed persistent hypocomplementemia despite an adequate disease management and absence of disease activity indicators over years. It should be mentioned that some of these patients maintained high double strand DNA levels despite SLE remission. As well, in some of these patients a diminution of more than one complement fraction was observed. These findings raise the doubt if, instead being consequence of PID, these conditions were related to a secondary complement deficit caused by persistence of autoantibodies directed against complement. Ideally, factor $\mathrm{C} 2$ should be quantified in these cases, given its prominent role in apoptotic debris removal [26]. Nevertheless, in Costa Rica no further complement fractions other than C1q, C3 and C4 are quantified in public hospitals.

Among patients with idiopathic neutropenia, a major association with autoimmune diseases was described anteriorly. Autoimmunity associated neutropenia occurs secondary to concomitant autoimmune disease, due to anti-polymorphonuclear antibodies. In several countries such as in Costa Rica, an important limitation for an accurate idiopathic neutropenia diagnosis is the unavailability of tests to determine anti-neutrophil antibodies, which are critical to differentiate between autoimmune neutropenia as a single entity or as a manifestation of other autoimmune diseases. The presence of antipolymorphonuclear antibodies excludes the diagnosis of idiopathic neutropenia, caused by various genetic alterations resulting in a decreased neutrophil count [27].

Regarding the pathogens isolated during infections, PID patients showed similarities with other case series published worldwide. In general, the organic complications mostly documented were respiratory ones. The relationship between IgG deficiency and pulmonary complications in PID is well known. Despite adequate replacement with IVIG, patients with PID continue to experience recurrent respiratory infections, triggering progressive lung function deterioration [28]. This clinical outcome was observed in patients of this study as well.

Unlike other countries in which subcutaneous immunoglobulin is routinely used for substitutive therapy, in Costa Rica only IVIG is available in the public health system so far. Unfortunately, this affects the adherence of our PID patients to treatment, increasing the negative impact of these conditions and worsening their prognosis.

Studies conducted over the past decade have corrected the underestimation about prevalence of PID in adults, evidencing that their occurrence is not lower than in children. Although most of these diseases are usually diagnosed in childhood, most PID patients are actually adults [21]. In any case, the management of PID differs significantly between children and adults, which requires an adequate, complex diagnostic approach, follow-up strategies and diverse therapeutic approaches [29]. The PID manifestations in adults can be multisystemic, therefore these disorders need an early suspicion and timely reference to a clinical immunologist specialized in adult patients, to prevent complications and sequelae. Similarly, the general population must be sensitized to suspect PID, so that patients show up early to the health system. 
Possibly, many adult carriers of PID remain undiagnosed worldwide, which limits truthful epidemiological knowledge about these entities in the general population. The promptness of the PID diagnosis is a crucial factor that can minimize the organic damage and therefore influence the prognosis directly. Likewise, early diagnosis of PID in adults is of high importance in terms of genetic counseling.

\section{Abbreviations}

APS-1 (autoimmune polyglandular syndrome type I), CVID (common variable immunodeficiency), IgA (immunoglobulin A), IgE (immunoglobulin E), IgG (immunoglobulin G), IgM (immunoglobulin M), IVIG (intravenous immunoglobulin), IU/mL (International standard Unit per milliliter), LOCID (late onset combined immunodeficiency), LASID (Latin American Association for Immunodeficiencies), PID (Primary immunodeficiency diseases), SIgAD (selective IgA deficiency), SLE (systemic lupus erythematosus), SPAD (specific polysaccharide antibody deficiency), XLS (X-linked agammaglobulinemia)

\section{Declarations}

\section{Funding}

No external financing or funding was available for this study.

\section{Conflicts of interest / competing interests}

The authors declare no conflict of interest and no competing interests in the development of this study or the derived publication.

\section{Availability of data and material}

The datasets generated and analyzed during the current study are available from the corresponding author on reasonable request. No other materials are available.

\section{Code availability}

Not applicable.

\section{Authorship contributions}

María del Rosario Espinoza Mora was responsible for the study conception and design. Data collection was performed by Alberto Alfaro Murillo and María del Rosario Espinoza Mora. Database design and analysis was performed by Christian Schauer. Statistical analyses were performed by Nayely Marquez Salazar and Milena Castro Mora. The first draft of the manuscript was written by María del Rosario Espinoza Mora and Christian Schauer. All authors commented on previous versions of the manuscript and all authors read and approved the final manuscript. 
Trial numberR017-SABI-00148, protocol approved $11 / 27 / 2017$ by the central ethic committee of the Caja Costarricense de Seguro Social. ClinicalTrials.gov: NCT04963660.

\section{Consent to participate}

Not applicable.

\section{Consent for publication}

Not applicable. In Costa Rica for submission no consent is required, a post-acceptance notification must be transmitted afterwards.

\section{References}

1. Cunningham-Rundles $C$. Autoimmunity in primary immune deficiency: taking lessons from our patients. Clin Exp Immunol. 2011;164(Suppl 2(Suppl 2):6-11.

2. Sánchez-Ramón S, Bermúdez A, González-Granado LI, et al. Primary and Secondary Immunodeficiency Diseases in Oncohaematology: Warning Signs, Diagnosis, and Management. Frontiers in immunology. 2019;10:586.

3. Driessen GJ, Dalm VA, van Hagen PM, et al. Common variable immunodeficiency and idiopathic primary hypogammaglobulinemia: two different conditions within the same disease spectrum. Haematologica. 2013;98(10):1617-23.

4. Liadaki K, Sun J, Hammarström L, et al. New facets of antibody deficiencies. Curr Opin Immunol. 2013;25(5):629-38.

5. Shah D, Romero F, Zhu Y, et al. C1q Deficiency Promotes Pulmonary Vascular Inflammation and Enhances the Susceptibility of the Lung Endothelium to Injury. J Biol Chem. 2015;290(49):29642-51.

6. Rosenberg E, Dent PB, Denburg JA. Primary Immune Deficiencies in the Adult: A Previously Underrecognized Common Condition. The Journal of Allergy Clinical Immunology: In Practice. 2016;4(6):1101-7.

7. Agarwal S, Cunningham-Rundles C. Autoimmunity in common variable immunodeficiency. Curr Allergy Asthma Rep. 2009;9(5):347-52.

8. Yazdani R, Habibi S, Sharifi L, et al. Common Variable Immunodeficiency: Epidemiology, Pathogenesis, Clinical Manifestations, Diagnosis, Classification, and Management. J Investig Allergol Clin Immunol. 2020;30(1):14-34.

9. Singh K, Chang C, Gershwin ME. IgA deficiency and autoimmunity. Autoimmun rev. 2014;13(2):16377.

10. Tichaczek-Goska D. Deficiencies and excessive human complement system activation in disorders of multifarious etiology. Adv Clin Exp Med. 2012;21(1):105-14.

11. McCusker C, Upton J, Warrington R. Primary immunodeficiency. Allergy Asthma Clin Immunol. 2018;14(Suppl 2):61. 
12. Condino-Neto A, Sorensen RU, Gómez Raccio AC, et al. Current state and future perspectives of the Latin American Society for Immunodeficiencies (LASID). Allergol Immunopathol. 2015;43(5):493-7.

13. Bousfiha A, Jeddane L, Al-Herz W, et al. The 2015 IUIS Phenotypic Classification for Primary Immunodeficiencies. J Clin Immunol. 2015;35(8):727-38.

14. Jesenak $M$, Banovcin $P$, Jesenakova $B$, et al. Pulmonary manifestations of primary immunodeficiency disorders in children. Front Pediatr. 2014;2:77.

15. Malesza IJ, Malesza M, Krela-Kaźmierczak I, et al. Primary Humoral Immune Deficiencies: Overlooked Mimickers of Chronic Immune-Mediated Gastrointestinal Diseases in Adults. Int J Mol Sci. 2020;21(15).

16. Kobrynski L, Powell RW, Bowen S. Prevalence and morbidity of primary immunodeficiency diseases, United States 2001-2007. J Clin Immunol. 2014;34(8):954-61.

17. Kindle G, Gathmann B, Grimbacher B. The use of databases in primary immunodeficiencies. Curr Opin Allergy Clin Immunol. 2014;14(6):501-8.

18. Marschall K, Hoernes M, Bitzenhofer-Grüber M, et al. The Swiss National Registry for Primary Immunodeficiencies: report on the first 6 years' activity from 2008 to 2014. Clin Exp Immunol. 2015;182(1):45-50.

19. Srinivasa BT, Alizadehfar R, Desrosiers $M$, et al. Adult Primary Immune Deficiency: What Are We Missing? The American Journal of Medicine. 2012;125(8):779-86.

20. Yazdani R, Azizi G, Abolhassani H, et al. Selective IgA Deficiency: Epidemiology, Pathogenesis, Clinical Phenotype, Diagnosis, Prognosis and Management. Scand J Immunol. 2017;85(1):3-12.

21. Thaventhiran JED, Lango Allen $\mathrm{H}$, Burren OS, et al. Whole-genome sequencing of a sporadic primary immunodeficiency cohort. Nature. 2020;583(7814):90-5.

22. Hamelmann E, Takeda K, Schwarze J, et al. Development of eosinophilic airway inflammation and airway hyperresponsiveness requires interleukin- 5 but not immunoglobulin $\mathrm{E}$ or B lymphocytes. Am J Respir Cell Mol Biol. 1999;21(4):480-9.

23. Wall LA, Dimitriades VR, Sorensen RU. Specific Antibody Deficiencies. Immunol Allergy Clin North Am. 2015;35(4):659-70.

24. Schmidt RE, Grimbacher B, Witte T. Autoimmunity and primary immunodeficiency: two sides of the same coin? Nat Rev Rheumatol. 2017;14(1):7-18.

25. Lintner KE, Wu YL, Yang Y, et al. Early Components of the Complement Classical Activation Pathway in Human Systemic Autoimmune Diseases. Frontiers in immunology. 2016;7:36.

26. Brodszki N, Frazer-Abel A, Grumach AS, et al. European Society for Immunodeficiencies (ESID) and European Reference Network on Rare Primary Immunodeficiency, Autoinflammatory and Autoimmune Diseases (ERN RITA) Complement Guideline: Deficiencies, Diagnosis, and Management. J Clin Immunol. 2020;40(4):576-91.

27. Palmblad J, Nilsson CC, Höglund P, et al. How we diagnose and treat neutropenia in adults. Expert Review of Hematology. 2016;9(5):479-87. 
28. Baumann U, Routes JM, Soler-Palacín P, et al. The Lung in Primary Immunodeficiencies: New Concepts in Infection and Inflammation. Frontiers in immunology. 2018;9:1837.

29. Grammatikos A, Bright $P$, Bhatnagar $R$, et al. How to investigate a suspected immune deficiency in adults. Respir Med. 2020;171:106100.

\section{Tables}

Table 1. General characteristics of patients and concomitant pathologies. Hospital

México and Hospital Dr. Rafael Angel Calderón Guardia, 1985-2018

\begin{tabular}{|c|c|c|}
\hline Sociodemographic and clinical features & Cases (n: 137) & $\%$ \\
\hline \multicolumn{3}{|l|}{ Age } \\
\hline 18 - 30 years old & 37 & 27.01 \\
\hline $30-59$ years old & 75 & 54.74 \\
\hline 60 years and older & 25 & 18.25 \\
\hline
\end{tabular}

Sex

$\begin{array}{lcc}\text { Female } & 84 & 61.31 \\ \text { Male } & 53 & 38.69\end{array}$

Place of origin

$\begin{array}{ccc}\text { Metropolitan area } & 118 & 86.13 \\ \text { Caribbean } & 6 & 4.38 \\ \text { Central and South Pacific } & 4 & 2.92 \\ \text { North Pacific } & 3 & 2.19 \\ \text { Foreigners } & 6 & 4.38\end{array}$

Nationality

$\begin{array}{lcc}\text { Costa Rican } & 131 & 95.62 \\ \text { Foreigners } & 6 & 4.38\end{array}$

Comorbidities

$\begin{array}{ccc}\text { Dyslipidemia } & 40 & 29.20 \\ \text { Acid peptic diseases } & 26 & 18.98 \\ \text { Hypertension } & 15 & 10.95 \\ \text { Diabetes mellitus 2 } & 6 & 4.38 \\ \text { Obesity } & 3 & 2.19 \\ \text { COPD } & 2 & 1.46\end{array}$

Note: Patients may have more than one comorbidity. Source: Own elaboration with data collected during the study. 
Table 2. Immunodeficiencies and clinical manifestations. Hospital México and Hospital Dr.

Rafael Angel Calderón Guardia, 1985-2018

\begin{tabular}{ccc}
\hline Sociodemographic and clinical features & Cases (n: 137) & $\%$ \\
\hline Type of immunodeficiency & 86 & 62.77 \\
Cellular & 33 & 24.09 \\
Other & 18 & 13.14 \\
Immunodeficiency manifestations & & \\
Recurrent infections & 81 & 59.12 \\
Atopic syndrome & 66 & 48.18 \\
Autoimmune disease & 41 & 29.93 \\
Neoplasia & 10 & 7.30 \\
Autoinflammatory syndrome & 4 & 2.92
\end{tabular}

Note: Patients may have more than one immunodeficiency manifestation. Source: Own elaboration with data collected during the study.

\section{Figures}




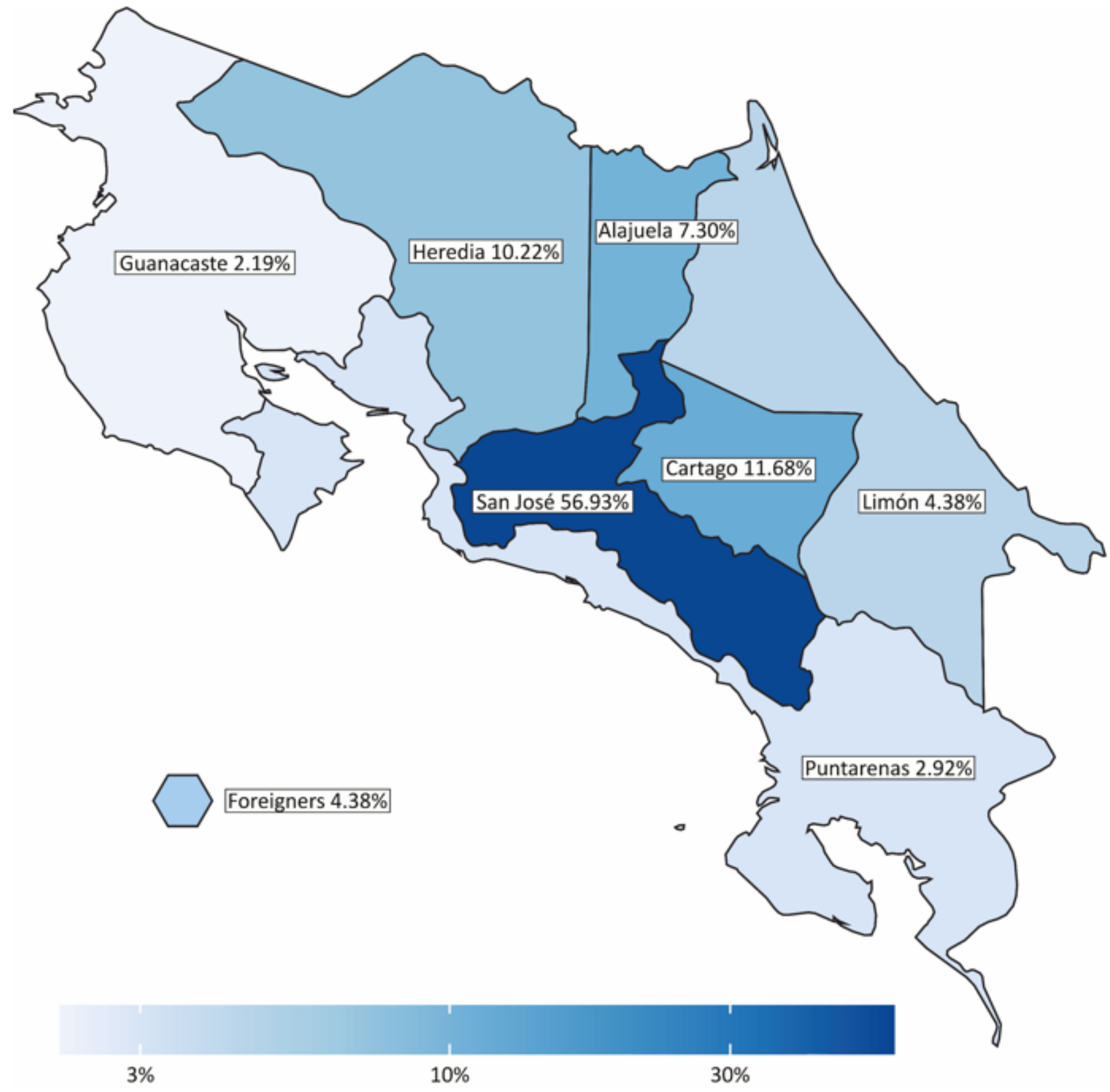

\section{Figure 1}

Percentage of adult patients with primary immunodeficiency diseases, attended at specialized clinics in Costa Rica from 1985 to 2018, considering the province of origin. Foreigners refer to patients not originally from Costa Rica (1 Cuban, 4 Nicaraguan, 1 nationality not registered). 
a)

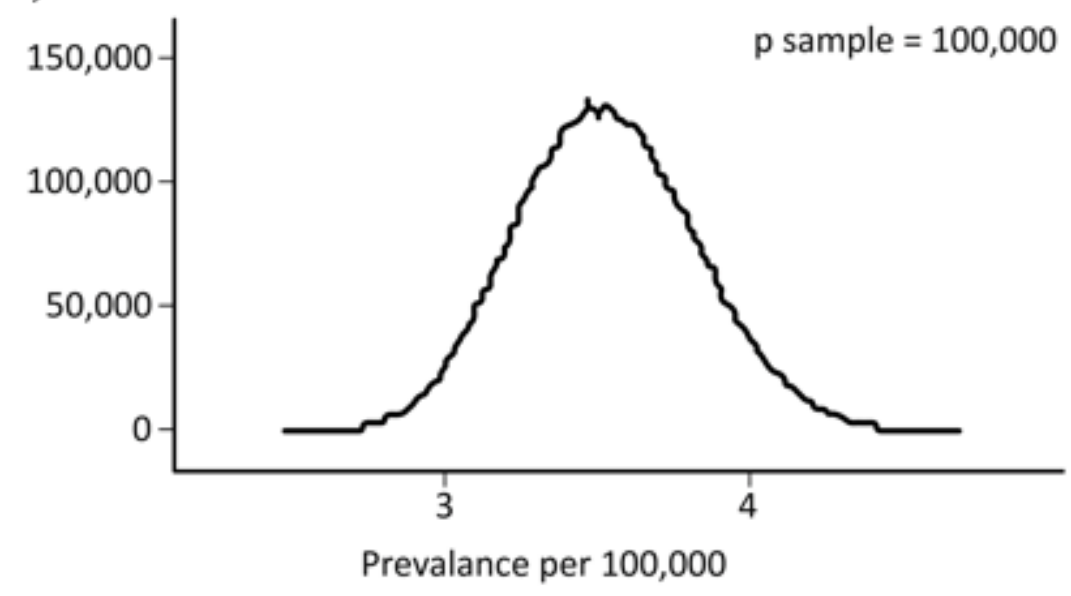

b)

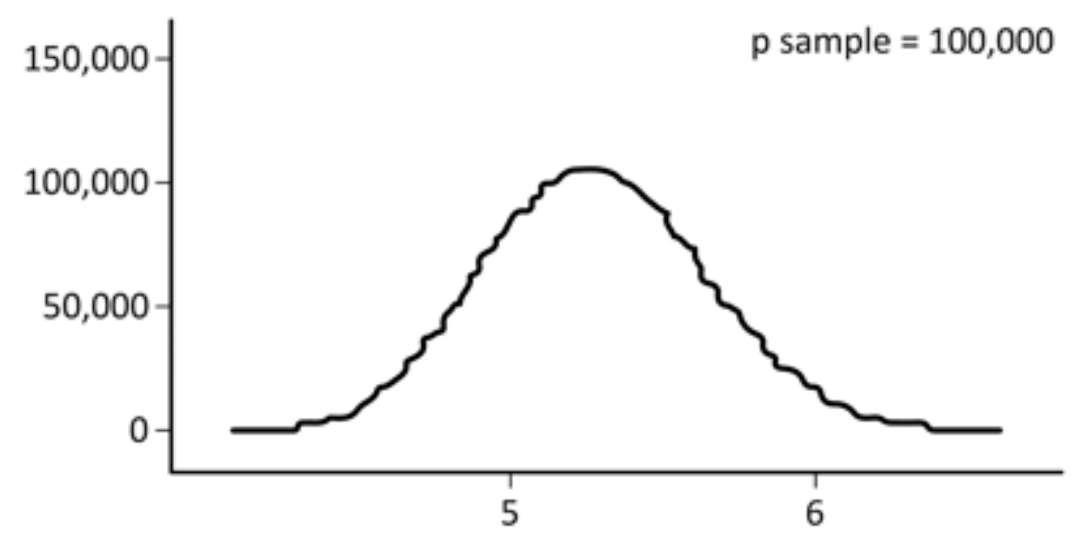

Prevalance per 100,000

Figure 2

Density of the posterior distribution. a) Model based in data of identified primary immunodeficiency disease patients, resulting in a mean prevalence of 3.35 patients per 100,000 inhabitants. b) Model considering a possible selection bias increase of prevalence posterior distribution of primary immunodeficiency dieseases considering a $50 \%$ of sample increment on the prevalence estimation, assuming additional undiagnosed patients are covered, resulting in a mean prevalence of 5.29 patients per 100,000 . 